\title{
Treatment of epistaxis without the use of nasal packing, a patient study*
}

\author{
Esther Vis and Herbert van den Berge
}

ENT Department, Medisch Centrum Leeuwarden, the Netherlands

\begin{tabular}{|l}
\hline SUMMARY \\
We questioned how many patients with epistaxis can be treated by cautery without the \\
use of nasal packing, as cautery is more effective and efficient. To investigate this, we \\
performed a retrospective study of a cohort of 418 patients with epistaxis who presented \\
to one ENT consultant at the ENT department of Medisch Centrum Leeuwarden (the \\
Netherlands) between 1997 and 2007. Main outcomes were the treatment modality (cau- \\
tery andlor nasal packing), recurrence of epistaxis and need for hospitalization. In $98 \%$ of \\
all patients the bleeding site could be found and treated by cautery. The incidence of recur- \\
rent bleeding was $6 \%$ Two percent of all patients had to be admitted to the hospital. This is \\
considerably lower compared with recurrence rates and hospitalization after treatment by \\
nasal packing known from the literature. Therefore we concluded that nearly all patients \\
can be effectively treated by cautery with a low recurrence rate. In addition, this method of \\
treatment is very cost effective. Because cautery requires skill and appropriate facilities, we \\
recommend special attention for this in ENT training programs. \\
Key words: etiology, epistaxis, therapy, cautery, nasal packing, recurrent bleeding, hospi- \\
talization
\end{tabular}

\section{INTRODUCTION}

Up to $60 \%$ of people experience epistaxis once in their lifetime, with $6 \%$ requiring medical attention ${ }^{(1)}$. The most important cause of epistaxis is idiopathic (70\%), followed by trauma and hypertension ${ }^{(2)}$. A nasal bleeding can be mild but can also lead to a potentially fatal situation, especially in the elderly using anticoagulants.

About 90 to $95 \%$ of all nasal bleeds originate from the anterior part of the nose, mainly from the Kiesselbach's plexus ${ }^{(3)}$. Posterior epistaxis mostly originates from the branches of the sphenopalatine artery and the anterior and posterior ethmoidal arteries. These bleeds can be severe and radical treatment such as clipping or embolization is sometimes necessary ${ }^{(4)}$.

With regard to the treatment of epistaxis, cautery is recommended as the treatment of first choice due to its efficiency and effectiveness ${ }^{(5-8)}$. Nasal packing is less effective because of a higher risk of recurrent bleeding and higher patient morbidity ${ }^{(9-13)}$. Moreover, nasal packing more often leads to hospitalization. Nasal packing is therefore recommended as second treatment of choice. In daily practice, however, $75 \%$ of all cases of epistaxis are treated by nasal packing and cautery is carried out in less than $20 \%{ }^{(14)}$. This fact is explicable by realizing that cautery can only be implemented when the bleeding point is visualized and can be reached with cautery instruments. This is time consuming and needs experience, skills and the accurate equipment ${ }^{(15)}$. On the other hand, packing a nose is a relatively easy and rapid method and therefore the treatment of choice of most general practitioners and emergency doctors. Nevertheless, also ENT specialists often use nasal packing in the daily management of epistaxis ${ }^{(16)}$.

Because of the effectiveness and efficiency of cautery, we questioned whether all patients with epistaxis presenting at our ENT department could be treated with cautery. Therefore we performed a study among patients presenting with a nasal bleed in which we tried to avoid the use of nasal packing. In addition, we studied the source of the bleeding (in children versus adults), the risk of recurrent bleeding, the admission rate, complications and predisposing factors such as age, use of anticoagulants, hypertension and other co morbidity.

\section{MATERIALS AND METHODS}

\section{Clinical treatment}

In the period between 1997 and 2007, 418 patients (213 male and 175 female; mean age 49 years) presenting with epistaxis treated by one ENT consultant were selected. This ENT consultant was in practice for eight years as an all-round nonacademic specialist at the beginning of the study. All patients presenting through the day and also in out of office hours when the consultant was on call were included. All patients 
were examined with a nose speculum and/or endoscope and microscope in an ENT treatment room with assistance of a nurse. Nasoendoscopy was done after local anesthesia on cotton wool pads soaked with lidocaine $10 \%$ and epinephrine 1:100.000. After identifying the bleeding site, cautery was performed using a hot wire $\left(\right.$ Erbe $\left.^{\circledR}\right)$ or silvernitrate $90 \%$.

\section{Data analysis set-up}

We distinguished active and non-active nasal bleeding. A nonactive bleed was defined as a bleed that has ceased before the moment of ENT consultation. The patient cohort was divided in patients younger than 18 years and older than 18 years to distinguish adults and children. For each patient, the age, localization of the bleed, the method of treatment, bleeding recurrence, complications of treatment, co-morbidity, use of medication, hospitalization and the length of hospitalization were documented. A recurrent bleed was defined as a new nasal bleed within a month after treatment, which resulted in a new (self-) referral to the hospital. To describe the localization of septal bleeds, we divided the septum of the nose into four zones numbered from I to IV (Figure 1). An anterior nasal bleed was defined as arising from zone I, II and the head of the concha inferior. A posterior nasal bleed was defined as arising from the septum zones III and IV and the corresponding areas on the lateral nasal wall, the concha media and the posterior part of the concha inferior.

\section{Statistical analysis}

SPSS version 16.0 was used.

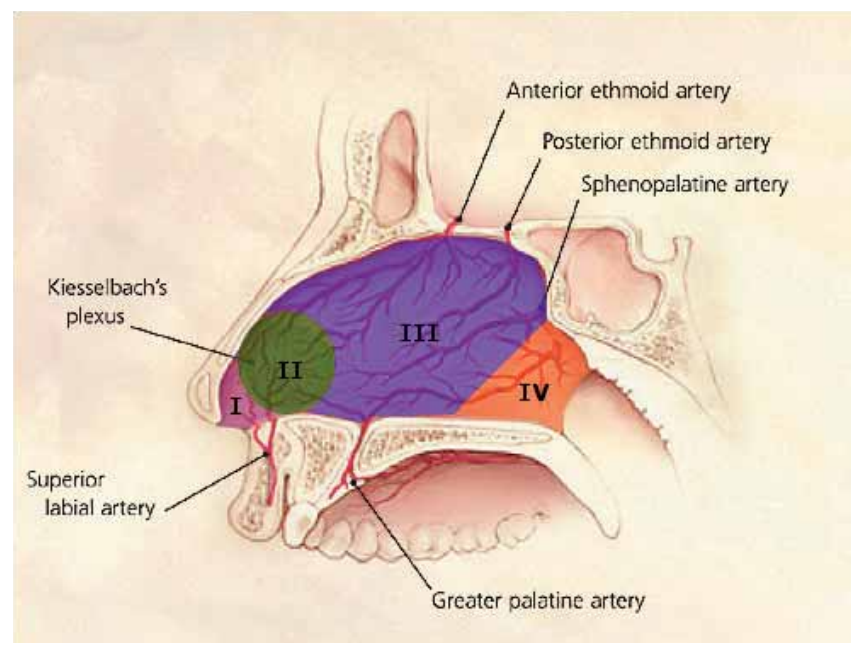

Figure 1. The nasal septum is divided into four zones: I to IV. Zone I: the most anterior part of the septum. Zone II: Kiesselbach's plexus. Zone III: part of the septum medial to the concha media and the origins of the anterior and posterior ethmoid artery and the entrance of the sphenopalatine artery. Zone IV: the most dorsal part of the septum.

\section{RESULTS}

\section{Treatment}

Table 1 presents the method of treatment of all patients with epistaxis. Of the 418 patients, 238 had a non-active bleed. Of this group, 137 patients were 18 years or older. One hundred thirty three $(97 \%)$ of them were treated with cautery, three $(2 \%)$ with local medical treatment and one $(1 \%)$ with nasal packing. This last patient was a female of 59 years old with a non-active recurrent bleed. Despite extensive nasoendoscopy, the bleeding area could not be identified.

Of all patients, 180 patients had an active bleed and 160 of them were older than 18 years. Of this group, 158 patients $(99 \%)$ were treated with cautery and two (1\%) with nasal packing. In these two patients, the bleeding area could not be identified.

\section{Localization of bleeding}

In patients younger than 18 years, the bleeding site was identified at the Kiesselbach's plexus in $97 \%$ in active bleeding and in $100 \%$ in non-active bleeding (Figure 2A and 2B). In patients older than 18 years with a non-active bleed, the bleeding site was located in $70 \%$ at the Kiesselbach's plexus, in $15 \%$ in zone III on the septum and in 10\% from one of the conchae (Figure 3A). In patients older than 18 years with an active bleed, the bleeding site was identified in $39 \%$ at the Kiesselbach's plexus, $36 \%$ in zone III and $12 \%$ was located on one of the conchae (Figure 3B).

One hundred fourteen patients had a posterior bleed. These

Table 1. Treatment of patients with epistaxis $(n=418)$.

\begin{tabular}{lcc}
\hline & $<18$ years & $>18$ years \\
\cline { 2 - 3 } $\begin{array}{l}\mathrm{n}=238 \\
\text { (non-active bleeding) }\end{array}$ & $101(100 \%)$ & $137(100 \%)$ \\
Cautery & $100(99 \%)$ & $133(97 \%)$ \\
Nasal packing & $0(0 \%)$ & $1(1 \%)$ \\
& $1(1 \%)$ & $3(2 \%)$ \\
Local medical treatment & & \\
$\mathrm{n}=180$ & $20(100 \%)$ & $160(100 \%)$ \\
(active bleeding) & & \\
Cautery & $20(100 \%)$ & $158(99 \%)$ \\
Nasal packing & $0(0 \%)$ & $2(1 \%)$ \\
Local medical treatment & $0(0 \%)$ & $0(0 \%)$ \\
\hline
\end{tabular}

Table 2. Recurrent epistaxis and hospital admission.

\begin{tabular}{lcc}
\hline & $\mathrm{n}=418$ years & $>18$ years \\
\cline { 2 - 3 } Recurrent bleeding (n total) & $121(100 \%)$ & $297(100 \%)$ \\
$\quad$ After non-active bleeding & $3(2.5 \%)$ & $23(7.7 \%)$ \\
After active bleeding & $3(2.5 \%)$ & $10(3.4 \%)$ \\
& 0 & $13(4.4 \%)$ \\
Hospital admission & & \\
(total + mean stay in days) & 0 & $10(1.3)$ \\
$\quad$ After non-active bleeding & 0 & $1(2)$ \\
$\quad$ After active bleeding & 0 & $9(1.2)$ \\
\hline
\end{tabular}


patients were successfully treated by cautery. Three hundred patients had an anterior bleed where $92 \%$ was localized at the Kiesselbach's plexus. Two hundred ninety seven patients $(99 \%)$ were treated with cautery and three with local medical treatment. Finally, there were four patients where no bleeding site could be identified. Three of them were treated with nasal packing and one with xylomethazoline $0.1 \%$ nasal drops.

\section{Recurrent bleeding}

Twenty six patients had recurrent bleeding and 23 of them $(88 \%)$ were initially treated by cautery. Three patients $(12 \%)$ were initially treated with nasal packing. Of all patients with recurrent bleeding, three patients $(12 \%)$ were younger than 18 years and $23(88 \%)$ older than 18 years. Thirteen patients $(50 \%)$ initially had active bleeding and the other 13 patients (50\%) non-active bleeding.

\section{Medication and co morbidity}

Three patients (1\%), all of them younger than 18 years, used nasal steroids for allergic rhinitis. One hundred fifty five patients (37\%), all of them older than 18 years, used anticoagu-

\section{Localization of bleeding in patients $<$ 18 years (non-active)}

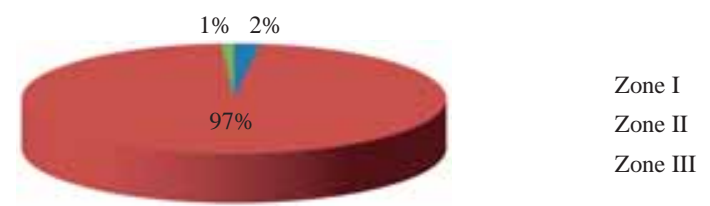

Figure 2A. Localisation of epistaxis in patients younger than 18 years with non-active nasal bleeding. The majority are localized in zone II (Kiesselbach's plexus).

\section{Localization of bleeding in patients > 18 years (non-active)}

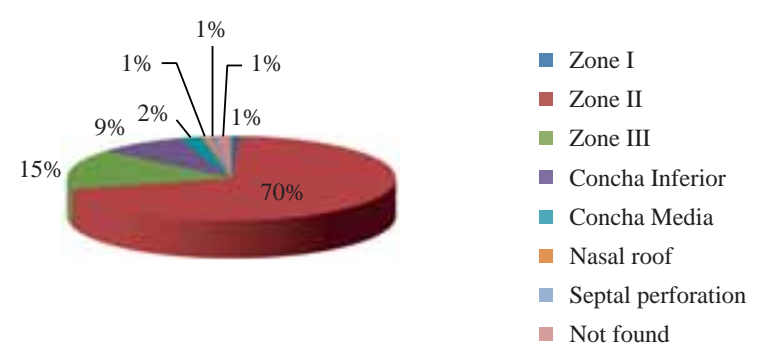

Figure 3A. Localisation of epistaxis in patients older than 18 years with non-active bleeding. $70 \%$ is located on the Kiesselbach's plexus. Subsequently bleeding sites are mostly located in zone III and the conchae nasalis media and inferior. lants. Their mean age was 73 years. Ten $(6.5 \%)$ of them had a proven elevated INR (International Normalized Ratio). Of the group of patients older than 18 years, 63 patients $(21 \%)$ were known with hypertension. Twelve (4\%) patients had a coagulation disorder caused by a hematological malignancy.

\section{Hospital admission}

Ten patients were admitted to the hospital. All of them were older than 18 years; their mean age was 75 years. Nine of them had active bleeding. All of them were treated by cautery. Three of them were admitted because of recurrent bleeding, two because of vasovagal reaction and one for treatment of a coagulation disorder and a blood transfusion. The remaining three were admitted for social reasons. The mean stay in the hospital was 1.2 days. Eight of the nine admitted patients were using anticoagulants of which three had an elevated INR. One patient (59 years old) had non-active bleeding and was admitted for two days. This patient was treated by nasal packing because the bleeding site could not be identified. Admission was necessary because of persistent bleeding.

\section{Localization of bleeding in patients $<$ 18 years (active)}

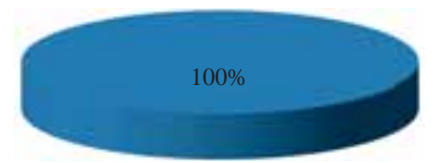

Zone II

Figure 2B. Localisation of epistaxis in patients younger than 18 years with active nasal bleeding. All bleeds are located at the Kiesselbach's plexus.

\section{Localization of bleeding in patients > 18 years (active)}
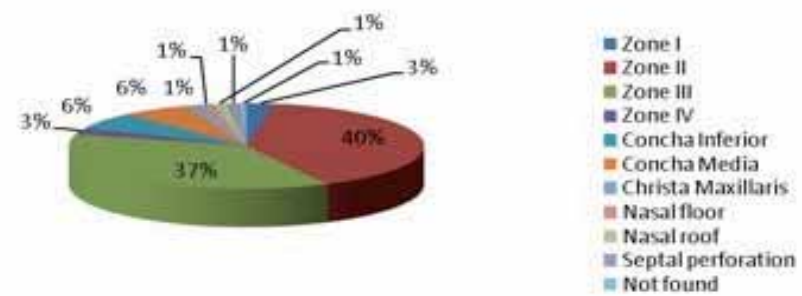

Figure 3B. Localisation of epistaxis in patients older than 18 years with active bleeding. $40 \%$ is located in zone II, $37 \%$ in zone III. $12 \%$ is located on one of the conchae nasalis. 


\section{Complications}

Three patients (mean age 79 years) had a vasovagal reaction during the treatment with cautery. This resulted in hospital admission of 2 days in two cases. One five year old patient fainted during treatment with cautery. Furthermore, one patient was seen for a second time because of recurrent bleeding from a septum perforation at the site of cautery, which was performed three weeks before. After a second cautery there was no recurrent bleeding anymore. Apart from that, the septum perforation did not give any symptoms.

Of all patients, four developed anemia as a result of the epistaxis. Three had been treated with cautery and one with nasal packing. One patient had an abnormally elevated INR and was treated with vitamin $\mathrm{K}$ and an infusion of saline solution. Another patient had a coagulation disorder. Eventually, one patient needed a blood transfusion.

\section{DISCUSSION}

In this study, we investigated whether all patients with epistaxis could be treated with cautery without using a nasal packing. Furthermore, we were interested in the localization of the bleeding, the incidence of recurrent bleeding, hospital admission rates, complications of treatment and predisposing factors. We were also interested in the influence of age and we questioned whether active versus non-active bleeding affected the outcome data mentioned above.

With regard to the treatment of epistaxis, it is known that although cautery is preferred, most patients are treated with a nasal packing ${ }^{(14)}$. A questionnaire among all ENT specialists in the Netherlands showed that $90 \%$ attempt to perform cautery but that only $8 \%$ uses cautery in all cases. Especially when presented with a bleed from the posterior nose, more than $25 \%$ of the specialists prefer treatment with nasal packing (16). The possibility of treating nearly every epistaxis with cautery was already described by Padgham in $1990^{(17)}$ and by Quine in $1994^{(7)}$ in 107 and 100 patients, respectively. We show that 411 of $418(98 \%)$ patients (children and adults) could effectively be treated by cautery without using nasal packing. This is a very high percentage compared to the treatment of epistaxis in daily ENT practice. Noticeable is that even in serious posterior epistaxis, we did not need to embolize or ligate the ethmoidal or sphenophalatine artery as described for example by Agreda ${ }^{(18)}$.

To achieve this, it is necessary to find the bleeding site. We showed that a greater number of all nasal bleeds (300 of 418, $72 \%)$ is localized on the anterior part of the nasal septum (Zone I-II, see Figure 1). A bleed from this part is relatively easily accessible for cautery when using a nasal speculum and/ or microscope. Posterior nasal bleeds occurred only in patients older than 18 years. In these cases, the bleeding site was often localized on the septum medial to the concha media $(66 \%)$. Also the concha media itself $(11 \%)$ and the entrance of the sphenopalatine artery in the area under the tail of the concha inferior (11\%) are relatively common bleeding sites. Therefore it is advisable to look at these sites first when identifying the site of a posterior bleed.

In all patients treated with cautery the chance of recurrent nasal bleeding within one month was 5.8\% (24 of 411 patients). In the literature, we could not find any data on recurrence rates after treatment with cautery. Van Wyk described a recurrence rate of $16 \%$ among patients with an uncomplicated nasal bleed treated with nasal packing, with recurrent bleeding within three days ${ }^{(14)}$. Faustauer even noted a recurrence rate of $37 \%{ }^{(19)}$. Obviously, treatment with cautery proved to have a considerably lower chance of recurrent bleeding as compared to nasal packing.

Besides the higher risk of recurrent bleeding, treatment with nasal packing more often leads to hospital admission. Ho even reported a percentage of $80 \%{ }^{(15)}$. Admission rates for cautery are much lower. Ahmed showed that $74 \%$ of the patients with epistaxis presenting at the emergency department treated with cautery could be treated on an outpatient basis ${ }^{(20)}$. Quine also described that treatment with cautery while performing microscopic examination leads to a reduction of hospital admission from $42 \%$ to $20 \%{ }^{(7)}$. In our study, the admission rate of all patients older than 18 years with an active bleed treated by cautery was as low as $5.6 \%$. Among patients with non-active bleeding this percentage was only $0.7 \%$. There was no difference between anterior and posterior bleeding. An explanation of these relatively low admission rates is that in nearly all patients, the bleeding site could be found and cautery solved the problem immediately, while we learned that the recurrence rate is very low. There was no need for any additional treatment. Knowing that an admission often takes more than 48 hours resulting in total costs of approximately 2000 Euro, treatment with cautery is not only more effective but also more efficient than nasal packing.

Apart from one patient who had a symptomless septal perforation after treatment with cautery, we found no complications associated with treatment with cautery. Nasal packing however, causes discomfort for the patient. In a recent study, patients assigned high pain scores to the introduction and removal of a Merocel tampon ${ }^{(9)}$. Common complications are sinusitis and (nocturnal) desaturation ${ }^{(10)}$. Less occurring but serious complications are the toxic shock syndrome ${ }^{(11)}$, pneumocephalus as a consequence of inaccurate placement of a tampon ${ }^{(12)}$ and a colon perforation as a result of swallowing the tampon (Rapid Rhino $\left.^{\circledR}\right)^{(13)}$.

To find out more about specific factors that can influence the occurrence of epistaxis and the seriousness of the bleeding, we studied age, co morbidity and use of medication. It was found that epistaxis often had a mild course and did not lead to any complications among patients younger than 18 years. On the contrary, epistaxis requires more attention among patients older than 18 years. Of this group, one out of five patients was known with hypertension. Moreover, $52 \%$ of these patients used anticoagulants, resulting in more serious bleeding. All 
these factors seem to play a role in the occurrence of epistaxis. However, an association with the occurrence of complications, anemia or recurrent bleeding was not found.

In conclusion, in this study we show that almost all patients with epistaxis can be treated with cautery without the use of nasal packing. Cautery is a very effective, efficient and safe method. Even in severe posterior bleeding, no additional treatment modalities such as embolization or ligation were necessary. In comparison with nasal packing, cautery results in less recurrent bleeding and hospital admission for anterior bleeds as well as for posterior bleeds. To achieve this, one should be well trained, have the proper equipment and accurate assistance.

\section{CONFLICT OF INTEREST STATEMENT}

The authors have no conflict of interest to report.

\section{REFERENCES}

1. Small M, Murray J, Maran AGD. A study of patiënts with epistaxis requiring admission to hospital. Health Bull (Edinb). 1982; 40: 20-29.

2. Iseh KR, Muhammad Z. Pattern of epistaxis in Sokoto, Nigeria: a review of 72 cases. Ann Afr Med. 2008; 7: 107-111.

3. Douglas R, Wormald PJ. Update on epistaxis. Curr Opin Otolaryngol Head Neck Surg. 2007; 15: 80-183.

4. Leppänen M, Seppänen S, Laranne L, Kuoppala K. Microcatheter Embolization of Intractable Idiopathic Epistaxis. Cardiovasc Intervent Radiol. 1999; 22: 499-503.

5. O'Donnell M, Robertson G, McGarry GW. A new bipolar diathermy probe for the outpatiënt management of adult acute epistaxis. Clin. Otolaryngol. Allied Sci. 1999; 24: 537-541.

6. Ahmed A, Woolford TJ. Endoscopic bipolar diathermy in the management of epistaxis: an effective and cost-efficient treatment. Clin Otolaryngol Allied Sci. 2003; 28: 273-275.

7. Quine SM, Gray RF, Rudd M, von Blumenthal H. Microscope and hot wire cautery management of 100 consecutive patiënts with acute epistaxis-a superior method to traditional packing. J Laryngol Otol. 1994; 108: 845-848.

8. Thornton MA, Mahesh BN, Lang J. Posterior epistaxis: identification of common bleeding sites. Laryngoscope. 2005; 115: 588-590.

9. Badran K, Malik TH, Belloso A, Timms MS. Randomized controlled trial comparing Merocel and RapidRhino packing in the management of anterior epistaxis. Clin Otolaryngol. 2005; 30:
333-337.

10. Jensen PF, Kristensen S, Juul A, Johannessen NW. Episodic nocturnal hypoxia and nasal packs. Clin Otolaryngol Allied Sci. 1991; 16: 433-435.

11. Hull HF, Mann JM, Sands CJ, Gregg SH, Kaufman PW. Toxic shock syndrome related to nasal packing. Arch Otolaryngol. 1983; 109: 624-626

12. Hollis GJ. Massive pneumocephalus following Merocel nasal tamponade for epistaxis. Acad Emerg Med. 2000; 7: 1073-1074.

13. Hashmi SM, Gopaul SR, Prinsley PR, Sansom JR. Swallowed nasal pack: a rare but serious complication of the management of epistaxis. J Laryngol Otol. 2004; 118: 372-373.

14. Van Wyk FC, Massey S, Worley G, Brady S. Do all epistaxis patients with a nasal pack need admission? A retrospective study of 116 patiënts managed in accident and emergency according to a peer reviewed protocol. J Laryngol Otol. 2007; 121: 222-227.

15. Ho EC, Chan J-Y. Front-line epistaxis management: let's not forget the basics. The Journal of Laryngology \& Otology. 2008; 122: 696-699.

16. Vis E, van den Berge H. The management of epistaxis in the Netherlands, the results of a questionnaire to ENT specialists. Abstracts of the bi-annual meeting of the Dutch ENT Society, 13-14 November 2008, Maastricht, The Netherlands. Clinical Otolaryngology. 2009; 34: 277.

17. Padgham N. Epistaxis: anatomical and clinical correlates. J Laryngol Otol. 1990; 104: 308-311.

18. Agreda B, Urpegui A, Ignacio Alfonso J, Valles H. Ligation of the sphenopalatine artery in posterior epistaxis. Retrospective study of 50 patients. Acta Otorrinolaringol Esp. 2011.

19. Faustauer M. Clinical outcome of patients with epistaxis treated with nasal packing after hospital discharge. Braz J Otorhinolaryngol. 2009; 75: 857-865.

20. Ahmed A, Woolford TJ. Endoscopic bipolar diathermy in the management of epistaxis: an effective and cost-efficient treatment. Clin Otolaryngol Allied Sci. 2003; 28: 273-275.

Esther Vis

Frouwesan 100

8939 EN Leeuwarden

The Netherlands

Tel: +31-58-844 3242/+31-61-865 8382

E-mail: esther.vis@znb.nl 